\title{
Interiorização da COVID-19 no Oeste da Bahia: perfil epidemiológico e análise espacial dos óbitos e casos confirmados
}

\author{
Inland expansion of COVID-19 in Western Bahia: epidemiological \\ profile and spatial analysis of deaths and confirmed cases
}

Daiene Rosa Gomes (https://orcid.org/0000-0002-1831-1259) ${ }^{1}$

Raiane Costa Souza (https://orcid.org/0000-0002-5037-249X) ${ }^{2}$

Uldérico Rios Oliveira (https://orcid.org/0000-0003-0465-1401) ${ }^{3}$

Mússio Pirajá Mattos (https://orcid.org/0000-0002-8792-5860) ${ }^{1}$

Ítalo Ricardo Santos Aleluia (https://orcid.org/0000-0001-9499-6360) ${ }^{1}$

Ana Maria Mapeli (https://orcid.org/0000-0002-6028-1989) ${ }^{4}$

${ }^{1}$ Núcleo de Pesquisa em Saúde Coletiva, Centro das Ciências Biológicas e da Saúde, Universidade Federal do Oeste da Bahia (UFOB).

R. Professor José Seabra de Lemos 316, Recanto dos Pássaros. 47808-021 Barreiras BA Brasil. daiene.gomes@ufob.edu.br ${ }^{2}$ Coordenadoria de Benefícios e Qualidade de Vida, UFOB. Barreiras BA Brasil.

${ }^{3}$ Departamento de Ciências Humanas, Universidade do Estado da Bahia. Barreiras BA Brasil.

${ }^{4}$ Centro das Ciências

Biológicas e da Saúde. UFOB. Barreiras BA Brasil.

\begin{abstract}
This article aims to describe the epidemiological profile and the spatial distribution of deaths and confirmed cases of COVID-19 in the health macroregion of Western Bahia. An ecological study on the inland expansion of SARS-CoV-2 was performed from March 21, 2020 to March 31, 2021 considering incidence and mortality rate, case fatality rate, case density and moving average of cases and deaths. 37,036 cases and 536 confirmed deaths were registered. Of all cases, $94.5 \%$ recovered and $4.0 \%$ remained active. The incidence rate was 3884.1/100,000 inhabitants, the mortality rate 56.2/100,000 inhabitants and the fatality rate was $1.4 \%$ A predominance of very high and high intensity of the occurrence of COVID-19 in the macroregion was identified and moving average revealed an increasing trend. Findings show a high risk of infection and death in the macroregion, in addition to a growing trend in accumulated cases, confirming the inland expansion of the disease.
\end{abstract}

Key words Coronavirus, Spatial Analysis, Epidemiology, Brazil
Resumo O objetivo deste artigo é descrever o perfil epidemiológico e a distribuição espacial dos óbitos e casos confirmados da COVID-19 na macrorregião de saúde Oeste da Bahia. Estudo ecológico sobre a interiorização do SARS-CoV-2, entre 21 de março de 2020 e 31 de março de 2021, considerando o coeficiente de incidência e de mortalidade, letalidade, densidade de casos e média móvel de casos e óbitos. Foram registrados 37.036 casos e 536 óbitos confirmados. Dos casos, 94,5\% estão recuperados e $4,0 \%$ ativos. O coeficiente de incidência foi 3.884,1/100 mil habitantes, o coeficiente de mortalidade, 56,2/100 mil habitantes, $e$ a letalidade, 1,4\%. Identificou-se a predominância de intensidade muito alta e alta da ocorrência da COVID-19 na macrorregião, com a média móvel evidenciando uma tendência de crescimento. Os achados descrevem alto risco de infecção $e$ morte na macrorregião, além de apresentar uma tendência de crescimento dos casos acumulados, confirmando a interiorização da doença.

Palavras-chave Coronavírus, Análise Espacial, Epidemiologia, Brasil 


\section{Introdução}

O novo coronavírus denominado SARS-CoV-2 foi identificado pela primeira vez na China em Wuhan (província de Hubei), em dezembro de $2019^{1}$, sendo considerado o agente causador da COVID-19 e que se disseminou rapidamente por todos os continentes. Diante disso, a Organização Mundial de Saúde (OMS) declarou que a doença apresenta uma emergência internacional de saúde pública ${ }^{2}$, caracterizada com o status de pandemia, em março de $2020^{3,4}$. Conforme a doença evolui, observa-se uma variedade de possibilidades na relação clínica, radiológica e laboratorial que pode levar a um dilema na condução de manifestações discordantes 5 . Por outro lado, possui características respiratórias predominantes que podem evoluir desde o desconforto respiratório à necessidade de cuidados intensivos ${ }^{6}$.

Nessa direção, a COVID-19 possui alta velocidade de disseminação e capacidade de provocar mortes, fatores que contribuem para o estado de incertezas sobre as melhores estratégias a serem adotadas no enfrentamento da pandemia em diferentes partes do mundo ${ }^{7}$. No Brasil, os desafios se apresentam mais latentes em virtude do cenário de grande desigualdade social, com populações vivendo em condições precárias de habitação e saneamento, sem acesso à água potável e em situação de aglomeração; além da extrema vulnerabilidade, com altas taxas de desemprego e cortes nas políticas sociais ${ }^{8}$.

Alguns estudos no país vêm sendo realizados para estimar a propagação da doença ${ }^{9-11}$, contudo, apesar da relevância dos achados, há que se considerar a heterogeneidade dos indicadores entre as diferentes regiões de saúde com transmissão, uma vez que esses variam de acordo com ações, rotinas, disponibilidade de suprimentos, estrutura de serviços de saúde e de vigilância, questões culturais e políticas ${ }^{12}$. Os casos e óbitos da COVID-19 já ocorrem nos grandes centros urbanos e no interior do país, sendo que Amazonas e Amapá, classificados como rurais e remotos, apresentaram incidência e mortalidade elevadas e colapso de seu sistema de saúde. A crença de que COVID-19 é "doença de cidade grande" cria obstáculos a mudanças de comportamento e prevenção, diante da urgência imposta pela pandemia em diferentes territórios ${ }^{13}$.

No Brasil, até o dia 31 de março de 2021 haviam sido confirmados 12.748 .747 casos de COVID-19 e 321.515 óbitos pela doença (2,5\% de letalidade $)^{14}$. Na Bahia, foram registrados 803.664 casos e 15.330 óbitos no mesmo período
$(1,9 \% \text { de letalidade })^{15}$. Além disso, a distribuição da COVID-19 tem ocorrido de forma heterogênea nas regiões de saúde, com os primeiros casos sendo identificados nas capitais brasileiras e, posteriormente, novos casos detectados em regiões mais afastadas, em detrimento da transmissão comunitária ${ }^{16}$.

Assim, existe uma tendência à interiorização da epidemia, o que desafia o controle da pandemia nas regiões mais remotas do país, dada a disseminação acelerada da COVID-19 em municípios de pequeno porte no país ${ }^{17}$. O conhecimento de como a doença está se distribuindo em diferentes regiões de saúde permite a compreensão de sua propagação. Nesse sentido, torna-se importante prever surtos regionais para desenvolver estratégias em resposta a emergência da COVID-19 em municípios do Nordeste brasileiro. Dessa forma, o presente estudo tem o objetivo de descrever o perfil epidemiológico e a distribuição espacial dos óbitos e casos confirmados da COVID-19 na macrorregião de saúde Oeste da Bahia.

\section{Métodos}

Trata-se de um estudo ecológico sobre a interiorização do SARS-CoV-2 na macrorregião de saúde Oeste da Bahia, a partir de 21 de março de 2020, data da confirmação do primeiro caso de COVID-19 na região, até 31 de março de 2021. Para tanto, utilizou-se dados públicos disponibilizados, diariamente, em boletins epidemiológicos das Secretarias Municipais de Saúde da região Oeste e da Central Integrada de Comando e Controle de Saúde do estado da Bahia (https:// bi.saude.ba.gov.br/transparencia/).

A macrorregião de saúde Oeste da Bahia é composta por 36 municípios (Figura 1A), abrange uma população de 953.520 habitantes e organizada em três regiões de saúde: a região de Barreiras, que abrange 15 municípios (Angical, Baianópolis, Barreiras, Brejolândia, Catolândia, Cotegipe, Cristópolis, Formosa do Rio Preto, Luís Eduardo Magalhães, Mansidão, Riachão das Neves, Santa Rita de Cássia, São Desidério, Tabocas do Brejo Velho e Wanderley), a de Ibotirama, com 09 municípios (Barra, Brotas de Macaúbas, Buritirama, Ibotirama, Ipupiara, Morpará, Muquém do São Francisco, Oliveira dos Brejões e Paratinga) e a de Santa Maria da Vitória, com 12 municípios (Bom Jesus da Lapa, Canápolis, Cocos, Coribe, Correntina, Jaborandi, Santa Maria da Vitória, Santana, São Félix do Coribe, Serra do Ramalho, Serra Dourada e Sítio do Mato ${ }^{18}$. 
Neste estudo foram analisadas as seguintes variáveis: número de casos confirmados, número de casos novos diários, número de óbitos confirmados, número de óbitos novos diários, casos ativos, casos recuperados, características demográficas (faixa etária, sexo, raça/cor e ocupação) e condições de saúde preexistentes. Os dados da população residente foram padronizados com os utilizados pela Secretaria de Saúde do estado da Bahia (SESAB) nos boletins epidemiológicos do estado para fins de comparação.

As frequências absolutas e relativas foram calculadas para os casos confirmados, que foram descritos segundo características demográficas e condições de saúde preexistentes. A partir do número absoluto de casos confirmados e óbitos por COVID-19, calculou-se o coeficiente de incidência (número de casos confirmados dividido pela população residente, multiplicado por 100.000 habitantes), coeficiente de mortalidade (número de óbitos por COVID-19 dividido pela população residente, multiplicado por 100.000 habitantes) e letalidade (número de óbitos por COVID-19 dividido pelo total de casos confirmados, multiplicado por 100). A média móvel semanal foi quantificada a partir da soma do número de casos e óbitos novos notificados nos últimos sete dias, dividido por sete (total de dias do período contabilizado), sendo considerado um cenário epidemiológico estável quando a variação percentual for de até $15 \%$; em crescimento, quando for maior que 15\%; e, em queda, quando for mais de $15 \%$ negativos.

Para a análise espacial, os dados foram processados e armazenados no Sistema de Informações Geográficas (SIG), por meio do software QGIS 2.18.28. Os arquivos vetoriais foram obtidos pelo IBGE $^{19}$ e Superintendência de Estudos Econômicos e Sociais da Bahia (SEI ${ }^{20}$. A partir da criação do Banco de Dados Geográfico (BDG) no SIG e análises no QGIS foram gerados os mapas dos casos confirmados, casos ativos, casos recuperados, número de óbitos, coeficiente de incidência, coeficiente de mortalidade, letalidade e densidade dos casos. Para a elaboração do mapa de densidade dos $\operatorname{casos}^{21}$ foi aplicada a técnica de mapa de calor, com raio de 30.000 metros, que possibilitou identificar os locais com maior densidade da ocorrência dos casos confirmados da COVID-19, por municípios da macrorregião estudada. Essa técnica proporciona a diferenciação dos municípios pela intensidade de ocorrência da COVID-19, sendo classificados como: muito alta e alta (municípios mais críticos), média (municípios em situação moderada) e baixa/muito baixa (municípios com menor intensidade de casos).
Os softwares Microsoft Excel for Windows, versão 2016, e Statistical Package for the Social Sciences (SPSS), versão 22.0, foram utilizados para tratamento, importação para BDG, análise de dados e criação de gráficos. O presente estudo foi realizado utilizando-se dados secundários e agregados, seguindo as recomendações das diretrizes da ética em pesquisa.

\section{Resultados}

No período estudado foram registrados $37.036 \mathrm{ca}-$ sos confirmados de COVID-19 na macrorregião de saúde Oeste da Bahia, sendo 61,5\% dos casos na região de Barreiras, $24,8 \%$ na região de Santa Maria da Vitória e 13,7\% na região de Ibotirama. Os municípios que registraram os maiores números absolutos de casos confirmados acumulados foram Barreiras $(28,2 \%)$ e Luís Eduardo Magalhães (16,3\%). Do total de casos confirmados na macrorregião, 94,5\% encontram-se recuperados e 4,0\% ativos, e, desses últimos, $49,1 \%$ situavam-se na região de saúde de Barreiras. A maior parte dos casos ativos registrados encontram-se nos municípios de Barreiras $(\mathrm{n}=304)$, Bom Jesus da Lapa ( $\mathrm{n}=172)$ e Luís Eduardo Magalhães $(\mathrm{n}=162)$, que juntos representam $42,8 \%$ dos casos ativos na macrorregião (Tabela 1, Figura 1B, 1C, 1D).

Com relação à faixa etária, houve maior prevalência de casos confirmados entre indivíduos de 30 a 39 anos (25,72\%). Quanto ao sexo, $54,08 \%$ dos casos eram do sexo feminino, $45,68 \%$ do sexo masculino e $0,24 \%$ não possuía informação. Em relação ao quesito raça/cor, verificou-se maior frequência da parda $(59,85 \%)$, seguida da branca $(14,36 \%)$ e amarela $(11,76 \%)$. No tocante a ocupação, $4,40 \%$ eram profissionais da saúde e concernente às condições de saúde preexistentes, destacaram-se a presença de doenças cardíacas crônicas (3,99\%); diabetes (2,00\%); doenças respiratórias crônicas descompensadas $(1,16 \%)$; imunossupressão $(0,30 \%)$; doenças renais crônicas em estágio avançado $(0,24 \%)$; doenças cromossômicas ou estado de fragilidade imunológica $(0,17 \%)$; houve o registro de apenas 1 caso de gestação de alto risco $(0,00 \%)$ (Tabela 2). É importante ressaltar que o quantitativo de casos descritos na Tabela 2 é inferior ao apresentado na Tabela 1, devido à indisponibilidade dos dados epidemiológicos detalhados e atualizados para todos os casos registrados nos municípios da macrorregião pesquisada.

O coeficiente de incidência da COVID-19 na macrorregião de saúde foi de 3.884,1/100 mil 

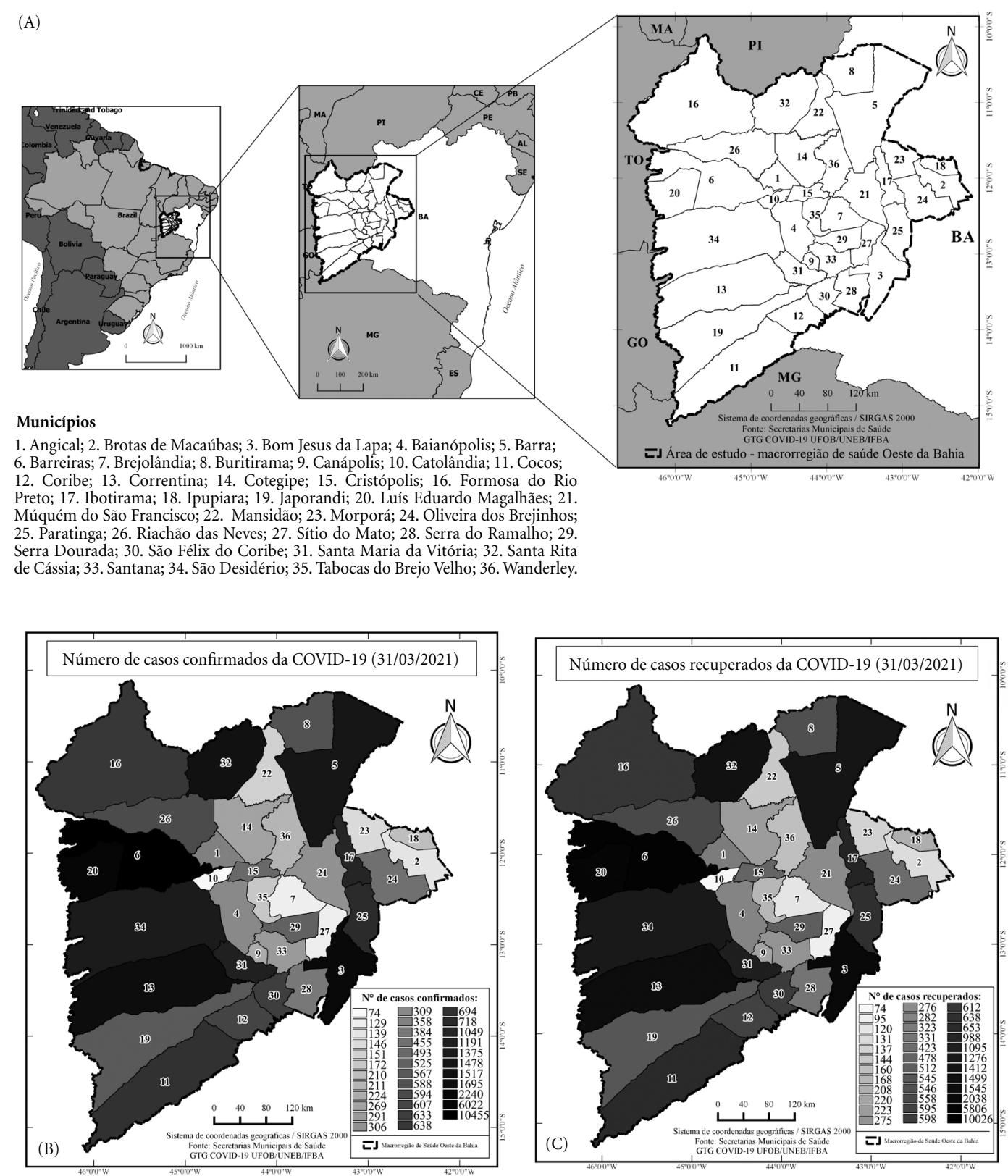

continua

Figura 1. Análise espacial dos casos e óbitos confirmados da Covid-19 na macrorregião de saúde Oeste da Bahia, até 31 de março de 2021. (A) Macrorregião de saúde Oeste da Bahia; (B) Casos confirmados; (C) Casos recuperados; (D) Casos ativos; (E) Óbitos.

hab, o coeficiente de mortalidade foi de 56,2/100 mil hab e a letalidade foi de $1,4 \%$ até o dia 31 de março de 2021. O maior coeficiente de incidência foi registrado na região de saúde de Barreiras (4.938,1/100 mil hab) e nos municípios de Luís Eduardo Magalhães (6.880,8/100 mil hab) e Barreiras (6.726,1/100 mil hab), ambos pertencentes à essa mesma região de saúde (Tabela 1, Figura $2 \mathrm{~A}, 2 \mathrm{~B}, 2 \mathrm{C})$.

O maior coeficiente de mortalidade foi registrado na região de saúde de Barreiras (62,2/100 mil hab) e no município de Jaborandi (119,3/100 mil hab), seguido dos municípios de Riachão das Neves (103,0/100 mil hab), Ibotirama (89,1/100 

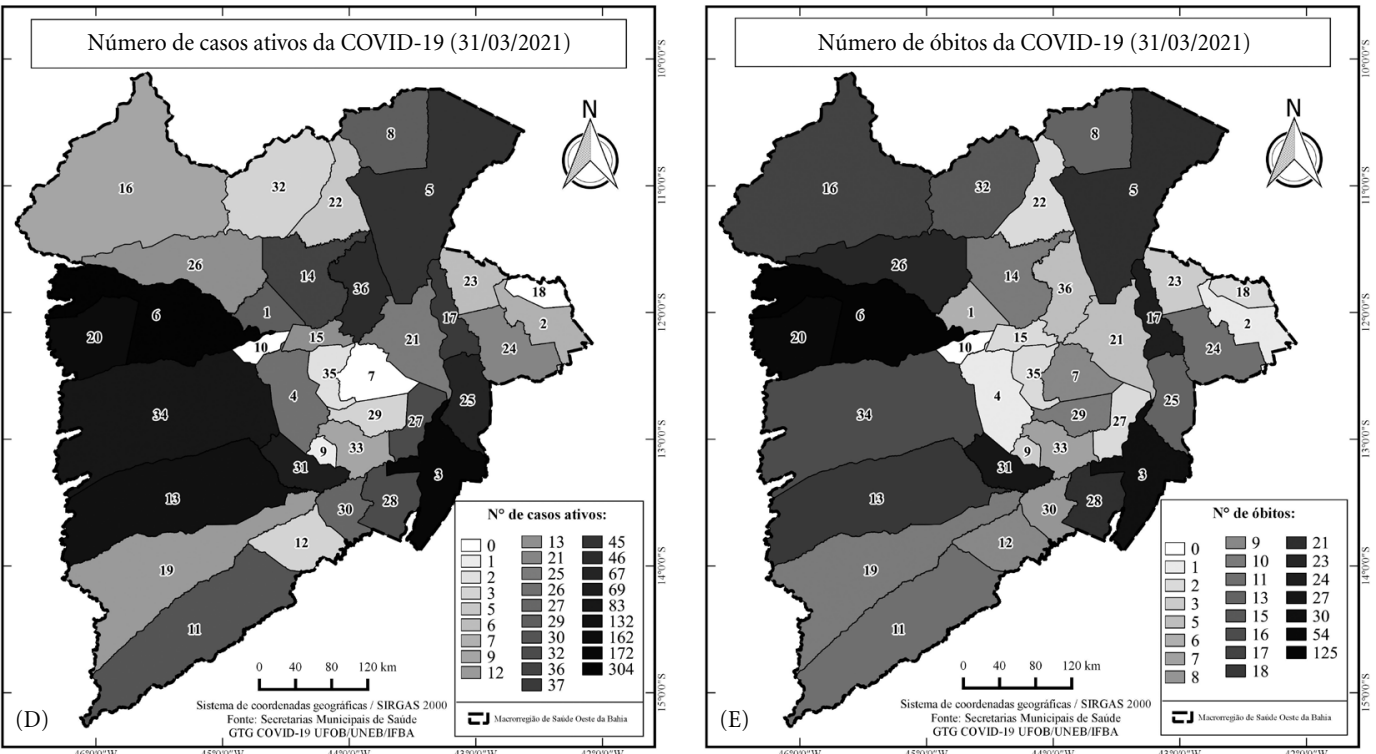

\section{Municípios}

1. Angical; 2. Brotas de Macaúbas; 3. Bom Jesus da Lapa; 4. Baianópolis; 5. Barra; 6. Barreiras; 7. Brejolândia; 8. Buritirama; 9. Canápolis; 10. Catolândia; 11. Cocos; 12. Coribe; 13. Correntina; 14. Cotegipe; 15. Cristópolis; 16. Formosa do Rio Preto; 17. Ibotirama; 18. Ipupiara; 19. Japorandi; 20. Luís Eduardo Magalhães; 21. Múquém do São Francisco; 22. Mansidão; 23. Morporá; 24. Oliveira dos Brejinhos; 25. Paratinga; 26. Riachão das Neves; 27. Sítio do Mato; 28. Serra do Ramalho; 29. Serra Dourada; 30. São Félix do Coribe; 31 . Santa Maria da Vitória; 32 . Santa Rita de Cássia; 33. Santana; 34. São Desidério; 35. Tabocas do Brejo Velho; 36. Wanderley.

Figura 1. Análise espacial dos casos e óbitos confirmados da Covid-19 na macrorregião de saúde Oeste da Bahia, até 31 de março de 2021. (A) Macrorregião de saúde Oeste da Bahia; (B) Casos confirmados; (C) Casos recuperados; (D) Casos ativos; (E) Óbitos.

Fonte: Secretarias Municipais de Saúde, GTG-COVID-19 UFOB/UNEB/IFBA.

mil hab), Brejolândia (85,3/100 mil hab) e Barreiras $(80,4 / 100$ mil hab). Dos cinco municípios, três estão localizados na região de saúde de Barreiras (Tabela 1, Figura 2C). Já com relação à letalidade, as maiores taxas foram observadas na região de saúde Ibotirama (1,8\%) e nos municípios de Brejolândia (7,0\%), Serra do Ramalho (5,5\%) e Riachão das Neves (3,9\%), sendo o primeiro e o último pertencentes à região de saúde de Barreiras e o segundo, à região de saúde de Santa Maria da Vitória (Tabela 1, Figura 2B).

A série histórica dos casos confirmados de COVID-19 mostra uma tendência crescente de casos, sendo registrado o maior valor absoluto no mês de dezembro (5.339 casos), seguido de agosto $(5.177$ casos) e março de 2021 (5.102 casos) (Figura 3A). Com relação ao quantitativo de municípios com notificação de casos confirmados, observa-se que houve um aumento significante, passando de dois no mês de março de 2020 (Barreiras e Barra) para trinta e seis $(100,0 \%)$, até o dia 31 de março (Figura 1B).

Com relação aos óbitos por COVID-19, foram confirmados 536 óbitos na macrorregião de saúde Oeste da Bahia, sendo que 287 (53,5\%) na região de saúde de Barreiras, 156 (29,1\%) na região de Santa Maria da Vitória e $93(17,4 \%)$ na região de Ibotirama. Os municípios que registraram os maiores números absolutos de óbitos foram Barreiras $(n=125)$ e Luís Eduardo Magalhães $(n=54)$, que juntos representaram 33,4\% dos óbitos (Tabela 1, Figura 1E).

Na série histórica de óbitos, verifica-se que o primeiro caso na macrorregião foi registrado no dia 10 de maio de 2020. Os maiores valores absolutos foram notificados no mês de agosto (92 óbitos), seguido de março de 2021 (83 óbitos) e setembro (64 óbitos) observou-se, também, um aumento considerável no quantitativo de municípios com notificação de ocorrência de óbitos, passando de um no mês de maio (Cristópolis) para trinta e cinco, até o dia 31 de março de 2021 (Figura 1E, 3B).

Quanto aos casos e óbitos novos, nota-se que no período analisado houve um aumento mais expressivo a partir do mês de julho, sendo a maior média mensal de casos novos registrada nos meses de dezembro (172 casos novos/ 


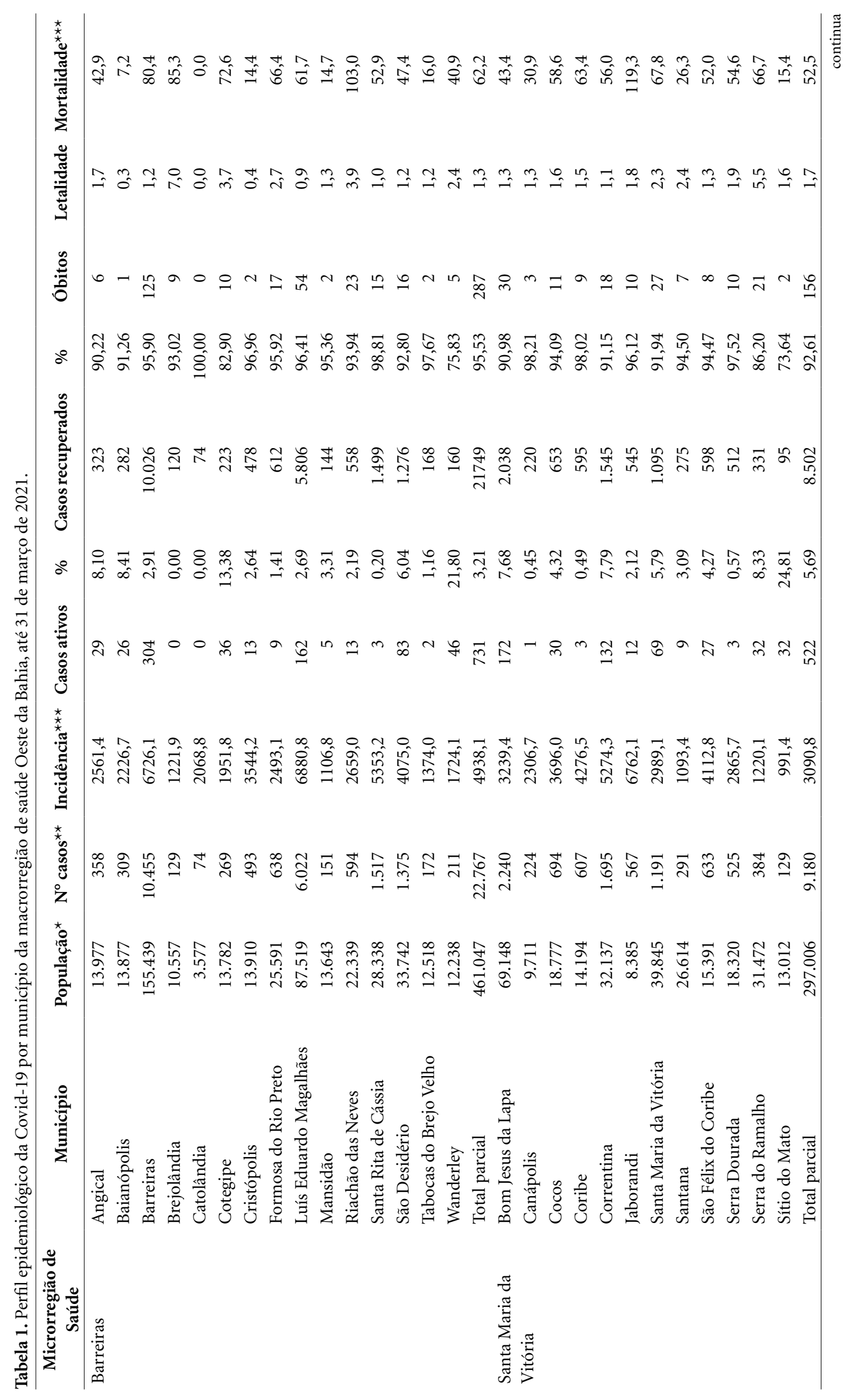




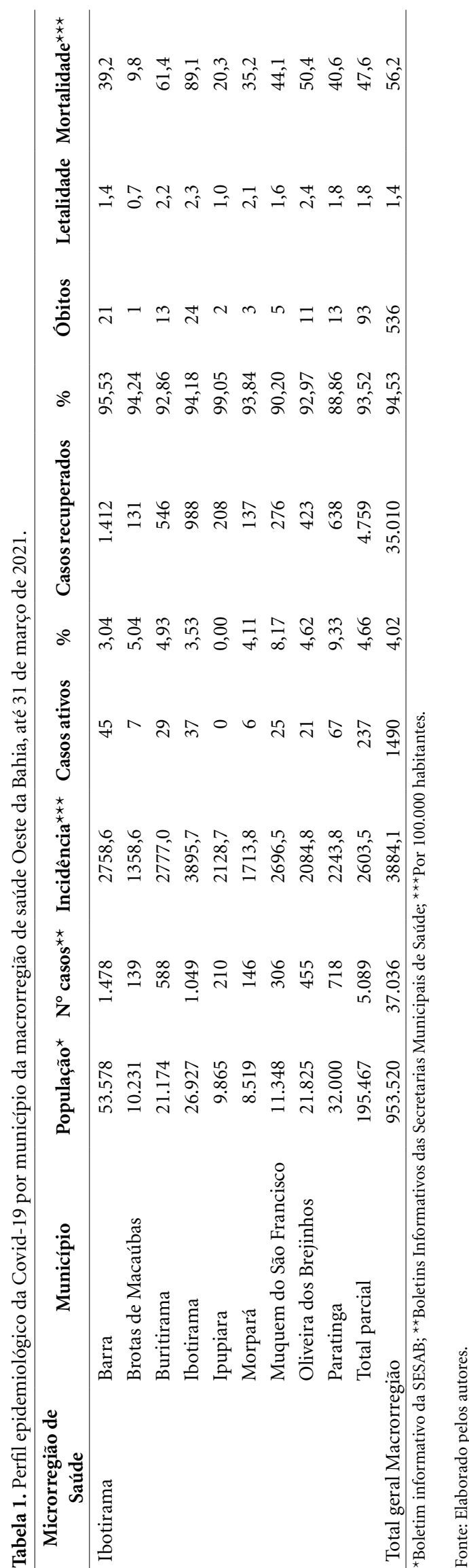

dia), seguido de agosto (167 casos novos/dia) e de março de 2021 (165 casos novos/dia) e a maior média mensal de óbitos, nos meses de agosto (3 óbitos novos/dia) e março de 2021 (3 óbitos novos/dia) (Figura 3A, 3B). Já com relação à média móvel de casos novos, nota-se oscilações importantes nos valores obtidos ao longo do período, sendo possível identificar picos nos meses de agosto (202 casos novos nos dias $07 \mathrm{e}$ 08/08/2020), dezembro (250 casos novos no dia 19/12/2020), mês no qual foi registrada a maior média móvel do período em análise, e março de 2021 (190 casos novos no dia 31/03/2021). Com relação à média móvel de óbitos por COVID-19, as oscilações nos valores foram menores, entretanto foi mantido comportamento similar ao de casos novos e os picos também foram registrados nos meses de agosto ( 4 casos novos nos dias 04 , 08, 09 e 23/08/2020), dezembro (4 casos novos nos dias 15 e 16/12/2020) e março de 2021 (4 casos novos no dia 31/03/2021). Quando avaliados os últimos 14 dias do período (18 e 31/03/2021), a média móvel de casos novos passou de 165 para 190 (aumento de $15,1 \%$ ) e a média móvel de óbitos novos de 2 para 4 (aumento de 50,0\%), o que evidencia, portanto, uma tendência de crescimento tanto no número de casos novos quanto de óbitos novos por COVID-19 na região (Figura $3 \mathrm{C}, 3 \mathrm{D})$.

Sobre a análise espacial dos casos confirmados, realizou-se um mapeamento da densidade desses casos, com vistas a compreender o padrão de distribuição espacial da COVID-19 na macrorregião de saúde Oeste da Bahia. Notou-se uma predominância de intensidade muito alta e alta da ocorrência da COVID-19 na macrorregião. Os municípios com a intensidade crítica de ocorrência foram representados pelo tom mais próximo ao preto (muito alta) e cinza escuro (alta), em situação moderada (Média), pelo tom mais próximo ao cinza médio e com menor intensidade de ocorrência no tom cinza claro (Baixa) e branco (Muito Baixa) (Figura 2D).

\section{Discussão}

Esse estudo analisou o perfil epidemiológico e a análise espacial da COVID-19 na macrorregião de saúde Oeste da Bahia. Com relação à incidência, o valor obtido pela macrorregião $(3.884,1 / 100$ mil hab) foi menor do que o registrado na Bahia $(5.403,5 / 100 \text { mil hab })^{15}$ e na maioria das suas demais macrorregiões de saúde, exceto a Norte $(3.721,2 / 100 \text { mil habitantes })^{15}$. Em contraparti- 
Tabela 2. Distribuição proporcional de casos confirmados da Covid-19 por características demográficas e condições de saúde preexistentes na macrorregião de saúde Oeste da Bahia, até 31 de março de 2021.

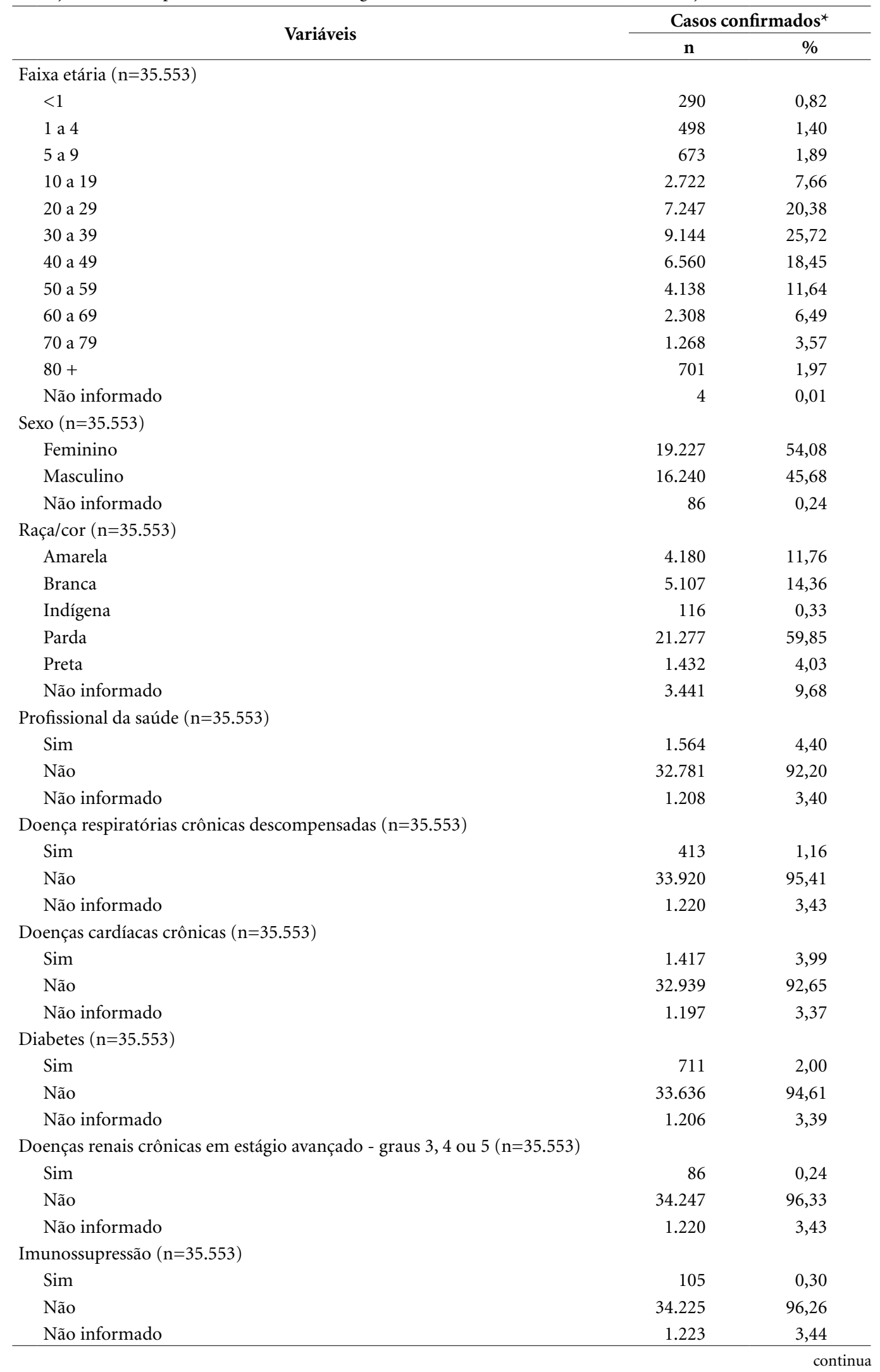


Tabela 2. Distribuição proporcional de casos confirmados da Covid-19 por características demográficas e condições de saúde preexistentes na macrorregião de saúde Oeste da Bahia, até 31 de março de 2021.

\begin{tabular}{lrr}
\hline \multicolumn{1}{c}{ Variáveis } & \multicolumn{2}{c}{ Casos confirmados $^{*}$} \\
\cline { 2 - 3 } & \multicolumn{1}{c}{$\mathbf{n}$} & \\
\hline Portador de doenças cromossômicas ou estado de fragilidade imunológica & & \\
$(\mathrm{n}=35.553)$ & 60 & 0,17 \\
Sim & 34.324 & 96,54 \\
Não & 1.169 & 3,29 \\
Não informado & & \\
Gestante de alto risco $(\mathrm{n}=35.553)$ & 1 & 0,00 \\
Sim & 34.383 & 96,71 \\
Não & 1.169 & 3,29 \\
Não informado & &
\end{tabular}

${ }^{\star}$ Dados obtidos pela Central Integrada de Comando e Controle de Saúde do estado da Bahia.

Fonte: Elaborado pelos autores.

da, a incidência da COVID-19 no Oeste baiano alcançou valores superiores aos registrados nos estados de Sergipe $(2.049,0 / 100$ mil hab), Paraíba $(1.713,0 / 100$ mil hab) e Ceará $(1.713,0 / 100$ mil hab) ${ }^{22}$, sendo inferior aos valores da região Nordeste $\left(4.994,4 / 100\right.$ mil hab ${ }^{23}$ e do Brasil $(5.898,5 / 100 \text { mil hab })^{23}$. Entretanto, os municípios de Barreiras e Luís Eduardo Magalhães obtiveram coeficientes de incidência maiores do que os registrados a nível estadual ${ }^{15}$, nos distintos estados do Sudeste e Nordeste ${ }^{23}$ e a nível nacional ${ }^{23}$.

A mortalidade acumulada na macrorregião de saúde alcançou um valor inferior ao registrado no estado da Bahia (103,0/100 mil hab) ${ }^{15}$, no Nordeste $(116,8 / 100 \text { mil hab })^{23}$ e no Brasil $(146,7 / 100 \text { mil hab })^{23}$. Entretanto, o município de Jaborandi apresentou destaque na mortalidade com valor acima do registrado no âmbito estadual ${ }^{15}$. Esse padrão não foi observado com a letalidade macrorregional, que registrou um valor menor que a Bahia $(1,9 \%)^{15}$, a região Nordeste $(2,3 \%)^{23}$ e o Brasil $(2,4 \%)^{23}$, todavia, nos municípios de Brejolândia, Cotegipe, Formosa do Rio Preto, Riachão das Neves e Serra do Ramalho, a letalidade foi superior ao encontrado a nível estadual $^{15}$, regional ${ }^{23}$ e nacional ${ }^{23}$. A mortalidade regional também superou valores registrados nos estados de Alagoas (43,3/100 mil hab), Paraíba (38,8/100 mil hab) e Piauí (35,4/100 mil hab) ${ }^{22}$.

Dos casos registrados, o sexo feminino predominou, seguindo o perfil da Bahia $(54,9 \%)^{15} \mathrm{e}$ da cidade do Rio de Janeiro $(51,4 \%)^{9}$, diferindo da média nacional $(45,1 \%)^{23}$. Os adultos jovens foram a faixa etária mais acometida, em concordância com o âmbito estadual $l^{15}$ e naciona ${ }^{23}$. Achados similares foram identificados em nível internaciona ${ }^{24}$, o que indica que a COVID-19 afeta principalmente a população economicamente ativa, entretanto, leva ao óbito os mais idosos e com condições de saúde preexistentes, como doenças crônicas e imunossupressão $0^{25}$, por isso a importância da vigilância e notificação desses dados. O presente estudo identificou uma baixa prevalência das condições de saúde preexistentes, com aproximadamente $3,4 \%$ dos casos sem esse tipo de informação, revelando fragilidades na notificação de informações relativas a um dos fatores de vulnerabilidade para a COVID-19.

No quesito raça/cor, predominou a parda, com quase $60,0 \%$ dos casos, apresentando valor superior ao observado na Bahia $(49,9 \%)^{15}$. No entanto, o Brasil obteve uma representação percentual inferior de pardos $(31,7 \%)^{23}$, apesar de ter o maior quantitativo de casos, possuindo prevalência da raça branca $(47,1 \%)$ e diferindo do padrão verificado na macrorregião de saúde Oeste. Sabe-se que as diferenças de saúde entre grupos raciais e étnicos são, geralmente, devido às condições econômicas e sociais ${ }^{26}$. Aliado a isso, o racismo também impede a adoção de medidas preventivas para COVID-19, considerando que o distanciamento social, a principal medida proposta pela $\mathrm{OMS}^{27}$, não é um privilégio de todos, em especial no Brasil, onde a população negra representa a maioria dos trabalhadores informais, de serviço doméstico, comercial, da alimentação, transporte, armazenamento e correio, que se mantiveram ativos, mesmo durante a pande$\mathrm{mia}^{28}$. Tal fato influencia o maior crescimento no número de infectados na população negra ${ }^{26}$.

No tocante à infecção da COVID-19 por profissionais da saúde, verificou-se um percentual 

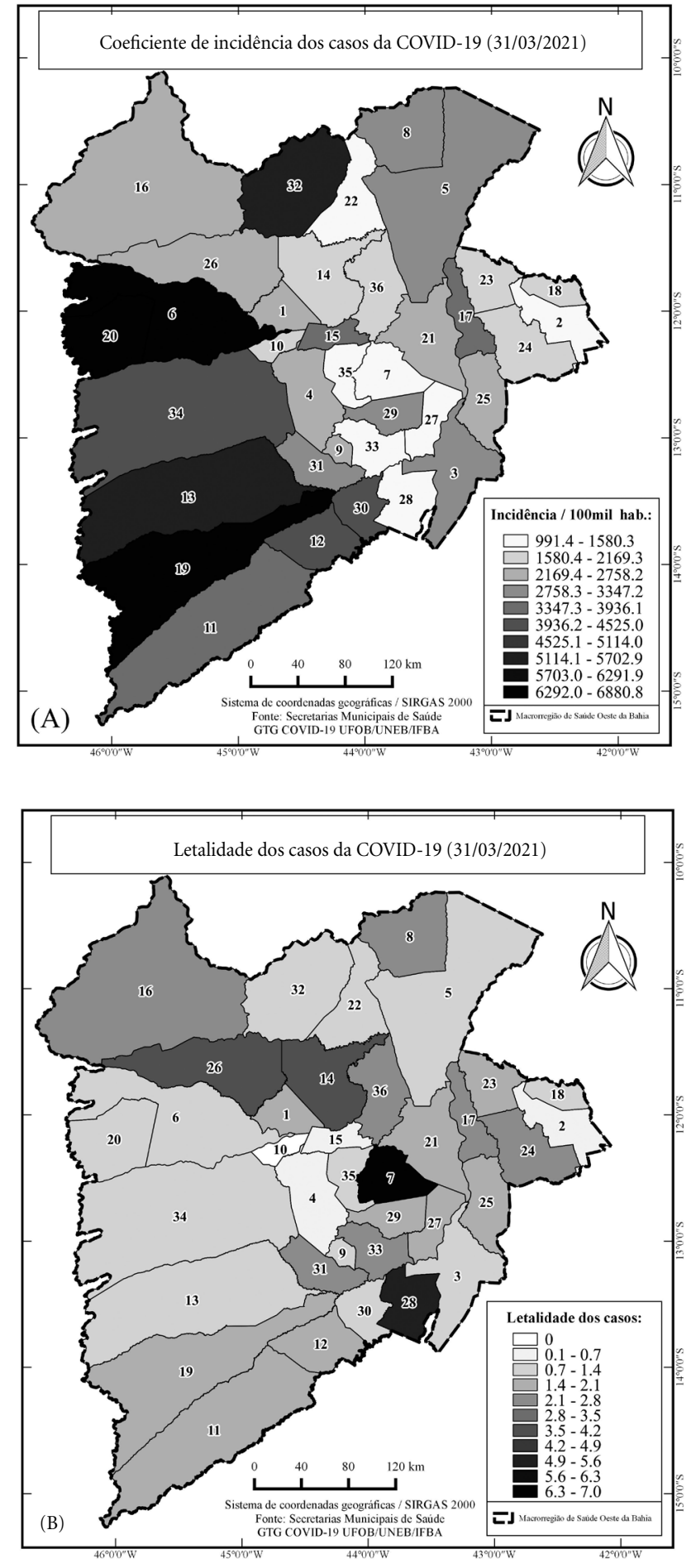

Municípios

2. Brotas de Macaúbas

3. Bom Jesus da Lapa

4. Baianópolis

5. Barra

6. Barreiras

7. Brejolândia

8. Buritirama

9. Canápolis

10. Catolândia

11. Cocos

12. Coribe

13. Correntina

14. Cotegipe

16. Formosa do Rio Preto

17. Ibotirama

18. Ipupiara

19. Japorandi

20. Luís Eduardo Magalhães

21. Múquém do São Francisco

22. Mansidão

23. Morporá

24. Oliveira dos Brejinhos

25. Paratinga

26. Riachão das Neves

26. Riachão das

28. Serra do Ramalho

29. Serra Dourada

30. São Félix do Coribe

31. Santa Maria da Vitória

33. Santana

34. São Desidério

35. Tabocas do Brejo Velho

36. Wanderley

Figura 2. Análise espacial da Covid-19 na macrorregião de saúde Oeste da Bahia, até 31 de março de 2021. (A) Coeficiente de incidência; (B) Letalidade dos casos confirmados; (C) Coeficiente de mortalidade; (D) Densidade dos casos confirmados. 
(C)
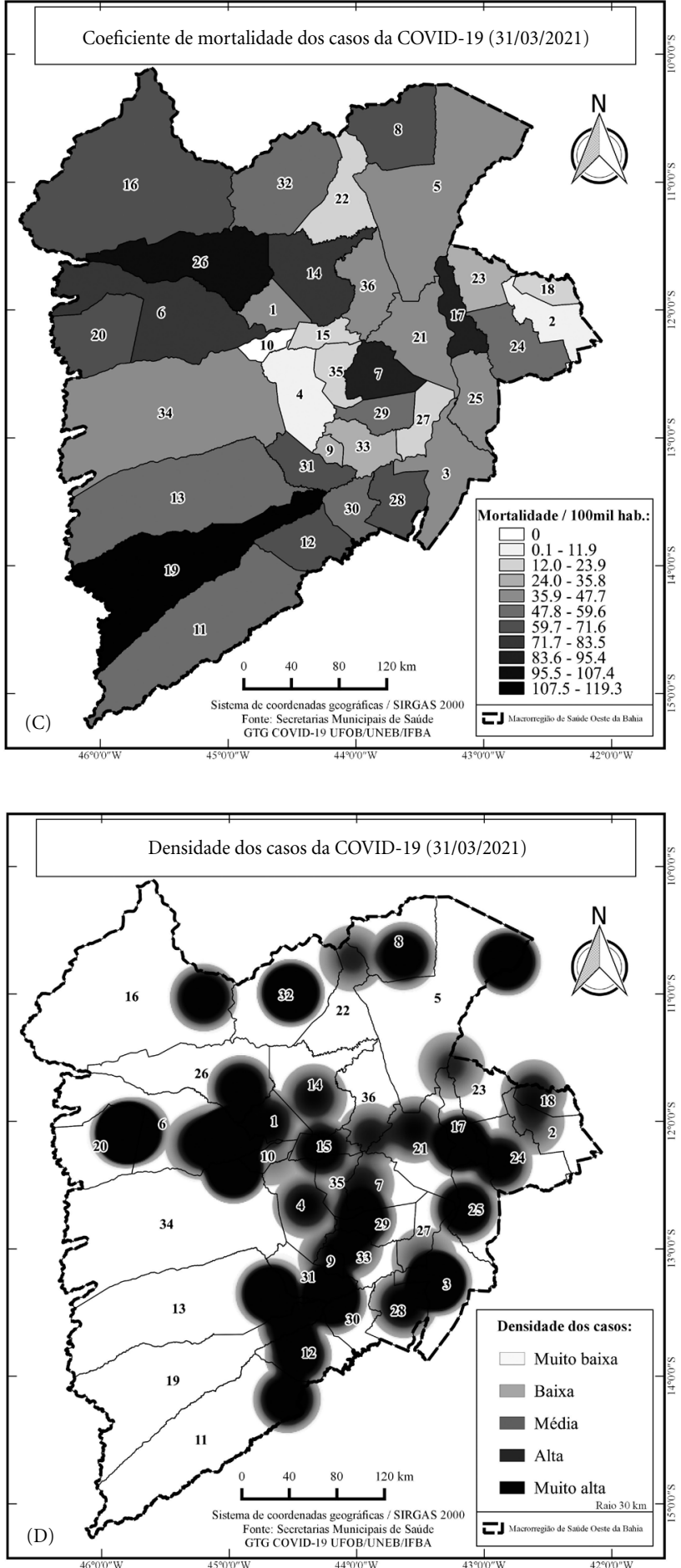

Municípios

1. Angical

2. Brotas de Macaúbas

3. Bom Jesus da Lapa

4. Baianópolis

5. Barra

6. Barreiras

7. Brejolândia

8. Buritirama

10. Catolândia

11. Cocos

12. Coribe

13. Correntina

14. Cotegipe

15. Cristópolis

16. Formosa do Rio Preto

17. Ibotirama

18. Ipupiara

19. Japorandi

20. Luís Eduardo Magalhães

21. Múquém do São Francisco

22. Mansidão

23. Morporá

24. Oliveira dos Brejinhos

25. Paratinga

26. Riachão das Neves

27. Sítio do Mato

28. Serra do Ramalho

29. Serra Dourada

30. São Félix do Coribe

31. Santa Maria da Vitória

32. Santa Rita de Cássia

33. Santana

34. São Desidério

35. Tabocas do Brejo Velho

36. Wanderley

Figura 2. Análise espacial da Covid-19 na macrorregião de saúde Oeste da Bahia, até 31 de março de 2021. (A) Coeficiente de incidência; (B) Letalidade dos casos confirmados; (C) Coeficiente de mortalidade; (D) Densidade dos casos confirmados. 


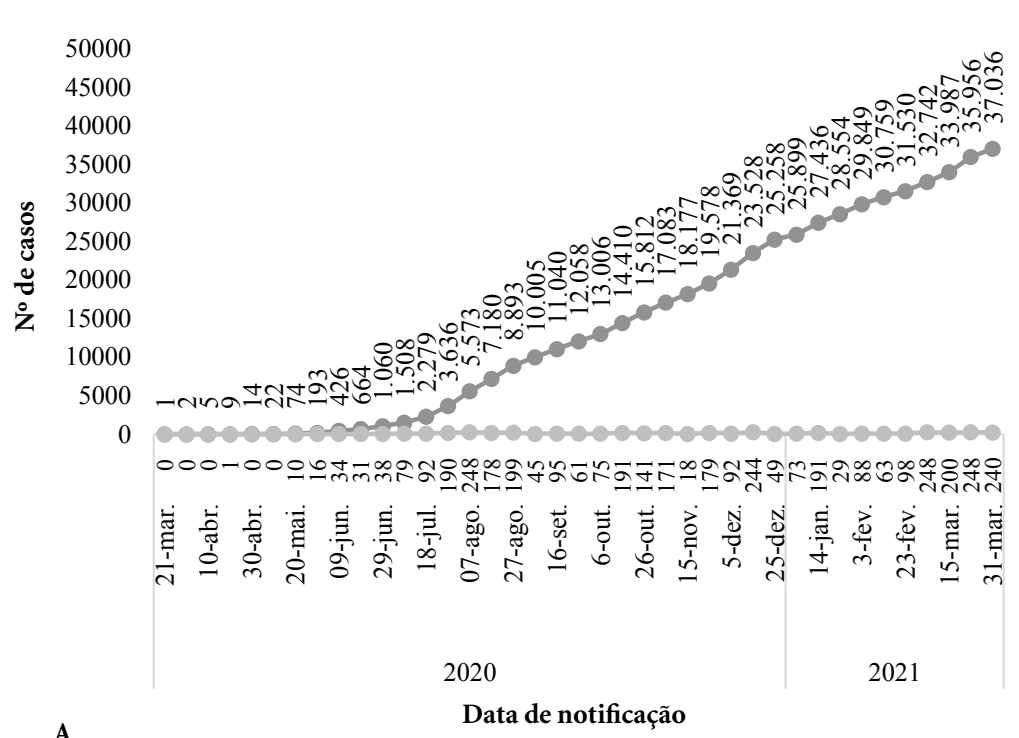

A
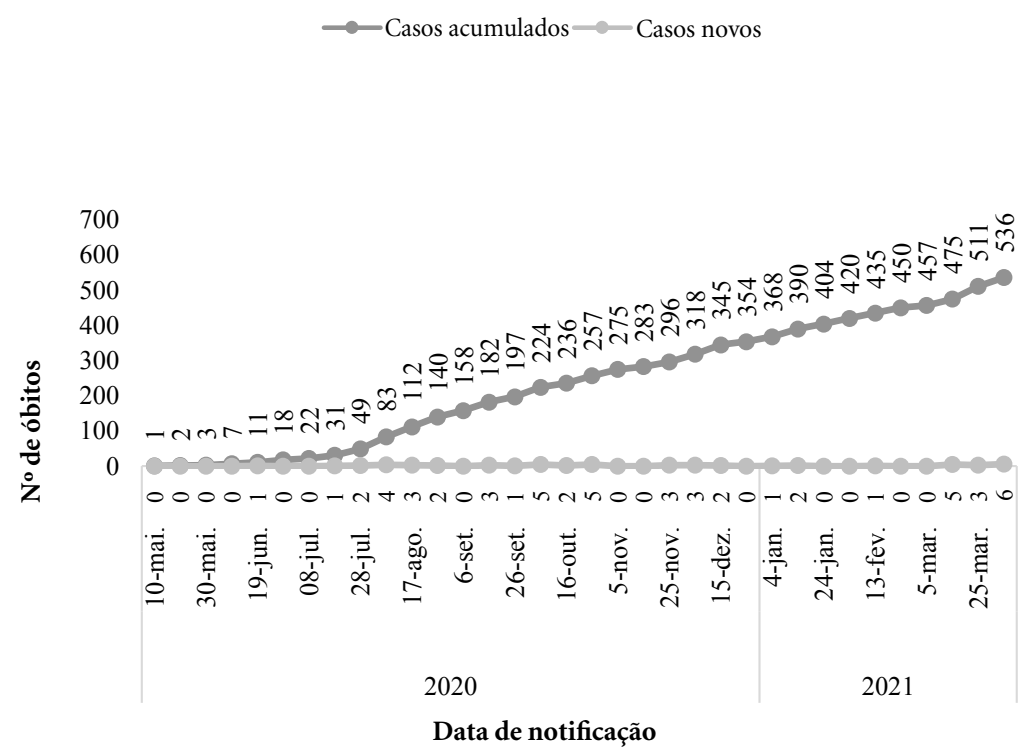

B

$\longrightarrow$ Óbitos acumulados $\longrightarrow$ Óbitos novos

continua

Figura 3. Série histórica de casos e óbitos confirmados e média móvel da Covid-19 nos municípios pertencentes à macrorregião de saúde Oeste da Bahia. até 31 de março de 2021. (A) Série histórica dos casos confirmados acumulados e novos; (B) Série histórica dos óbitos confirmados acumulados e novos; (C) Casos novos confirmados e média móvel; (D) Óbitos novos confirmados e média móvel.

menor do que o registrado na Bahia $(5,85 \%)^{15}$. Segundo boletim da SESAB sobre os trabalhadores em saúde, os mais acometidos têm vínculo empregatício terceirizado e, dentre as categorias de nível superior, destacam-se: enfermeiras(os) $(17,2 \%)$, auditores $(16,7 \%)$ e aqueles em cargos de direção, coordenação e chefia nas unidades (15,5\%). Já entre as categorias de nível técnico, destacaram-se os(as) técnicos(as)/auxiliares de laboratório/patologia (20,6\%), seguidos pelos técnicos(as)/auxiliares de enfermagem (19,0\%) e os/ as técnicos(as)/auxiliares odontologia $(14,7 \%)^{29}$. 

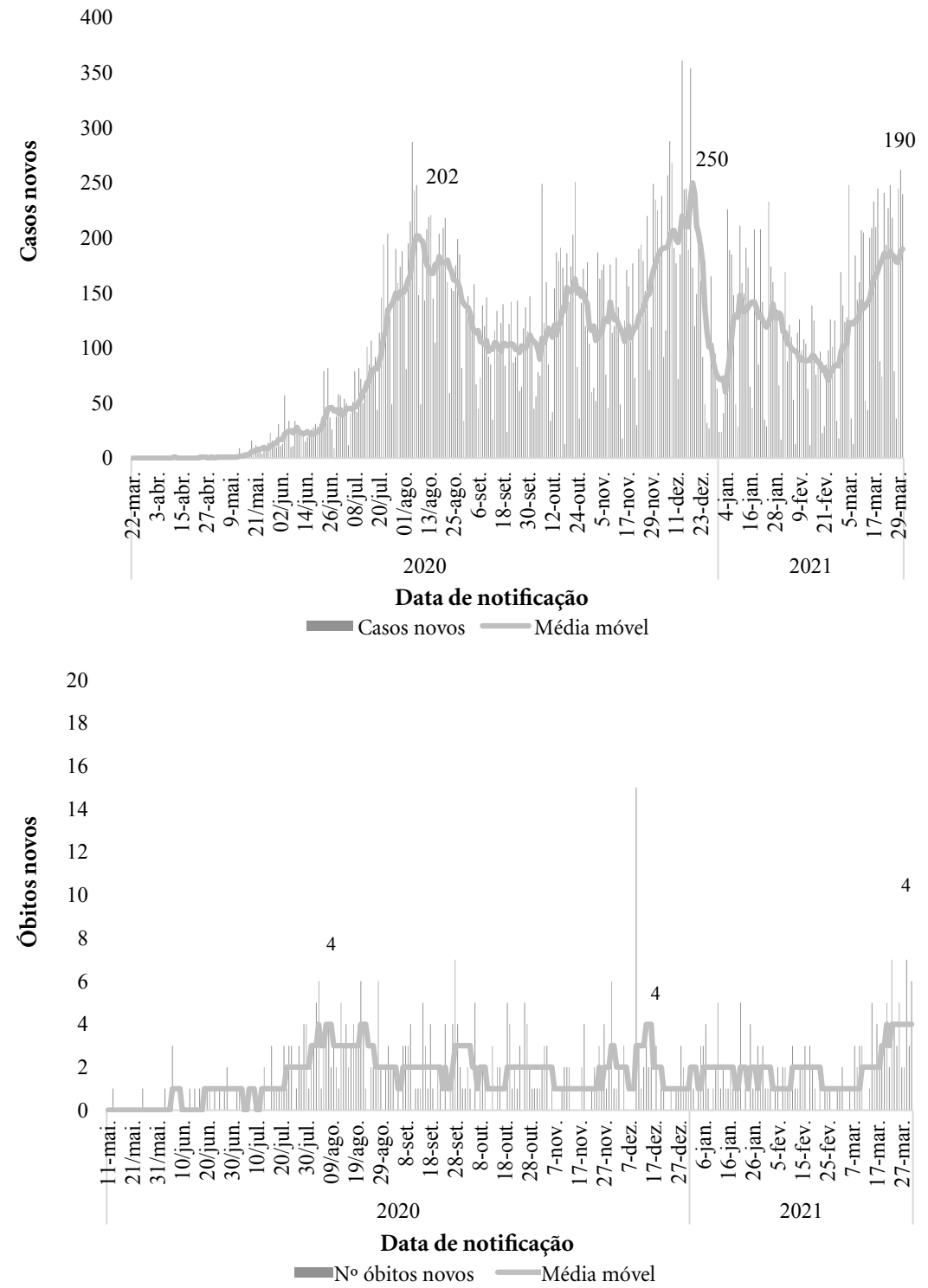

Figura 3. Série histórica de casos e óbitos confirmados e média móvel da Covid-19 nos municípios pertencentes à macrorregião de saúde Oeste da Bahia. até 31 de março de 2021. (A) Série histórica dos casos confirmados acumulados e novos; (B) Série histórica dos óbitos confirmados acumulados e novos; (C) Casos novos confirmados e média móvel; (D) Óbitos novos confirmados e média móvel.

Fonte: Elaborado pelos autores.

A evolução da COVID-19 ocorreu de forma distinta entre os municípios, sendo inicialmente registrados casos confirmados em apenas dois deles, em março de 2020, após um ano, constatou-se casos confirmados nos 36 municípios da macrorregião de Saúde Oeste. Com relação aos casos recuperados, observa-se que a macrorregião apresentou um percentual inferior ao relatado na Bahia $(96,2 \%)^{15}$ e maior comparado ao verificado no Brasil $(87,1 \%)^{23}$. Ressalta-se que devido à indicação de coleta de exames para confirmação diagnóstica de infecção pelo novo coronavírus ser restrita a públicos definidos pelos protocolos do Ministério da Saúde e protocolos municipais e, consequentemente, ocorrência de subnotificação de casos, é necessário avaliar com cautela esses dados sobre proporção de recuperados versus casos ativos. 
O primeiro caso de COVID-19 confirmado na macrorregião ocorreu em Barreiras, município com o maior número de casos acumulados. Trata-se de um dos maiores polos agropecuários baianos e do Nordeste brasileiro, o que dinamiza e influencia a economia das regiões de saúde. Em 2017, situava-se entre os municípios com o maior PIB per capita da Bahia, R $\$ 24.676,48^{30}$. Ainda, este município é o único que possui aeroporto com voos para diversas cidades brasileiras, além de ser considerado o principal entroncamento rodoviário da região, o que acarreta grande fluxo de pessoas por meio rodoviário e aéreo, oferecendo mobilidade para regiões próximas e a outros estados brasileiros, o que pode estar relacionado com a transmissibilidade da doença. $O$ fluxo de turistas para região na alta temporada, como feriados de Natal, Ano Novo, Carnaval e São João, também pode ter contribuído para a entrada do vírus no território e sua propagação. Embora os primeiros casos de COVID-19 tenham sido registrados em março, provavelmente a contaminação tenha ocorrido muito tempo antes, devido ao período de grande corrente de turistas em aeroporto, rodoviárias e espaços com aglomeração de pessoas ${ }^{31}$.

No que se refere à média móvel de casos e óbitos registrada no último dia de observação do período de estudo (31 de março de 2021), observa-se uma tendência de crescimento na ocorrência de casos novos e óbitos novos. Entretanto, considerando que o cenário epidemiológico é influenciado diretamente pelas medidas de controle adotadas e que pode sofrer alterações ao longo do tempo, a condição de crescimento pode estar associada ao relaxamento das medidas de prevenção da COVID-19 nos territórios municipais.

Com a densidade dos casos confirmados foi possível compreender o padrão de distribuição espacial da COVID-19 na macrorregião de saúde Oeste da Bahia. Esse mapeamento permitiu discriminar os locais com maior densidade na ocorrência dos casos. Essa identificação sinaliza a necessidade de intensificação das medidas de combate à doença, a exemplo das medidas de distanciamento social e de vigilância epidemiológica e sanitária, para que seja possível a prevenção da disseminação de novos casos nestes municípios.
Além disso, faz-se necessária uma abordagem de enfrentamento à COVID-19 de forma coordenada no âmbito intra e intermunicipal, considerando a proximidade e fluxo de indivíduos entre as cidades da região.

Algumas limitações são importantes na interpretação dos achados deste estudo. Os casos confirmados estão relacionados predominantemente a critérios laboratoriais. Deste modo, revela-se uma potencial subnotificação no território estudado, principalmente pela existência de apenas um Laboratório de Saúde Pública regionalizado. Os coeficientes de incidência e mortalidade podem não refletir bem as informações para os municípios com a população pequena, como Catolândia e Cotegipe, que apresentam taxas elevadas. De modo semelhante, encontrase municípios com número pequeno de casos e óbitos, como Brejolândia e que possui 7,0\% de letalidade.

Os achados indicam alto risco de infecção e morte pela doença em municípios da macrorregião de saúde Oeste da Bahia. Além disso, evidenciam uma tendência de crescimento de casos novos, confirmando o perfil nacional de interiorização da COVID-19 nas cidades menores. Admite-se que a distribuição pode se alterar rapidamente, devido à transmissão e características das intervenções municipais, estrutura dos serviços de saúde, aspectos socioeconômicos, culturais e políticos.

Entretanto, reafirma-se que a interiorização gradativa da doença pode afetar ainda mais o sistema de saúde da macrorregião, dado que muitos municípios não têm sequer hospital, sendo necessário o deslocamento para o município de referência da regional de saúde. Dessa forma, recomenda-se a intensificação das medidas de prevenção orientadas pelas autoridades sanitárias, especialmente nos municípios que apresentaram os maiores coeficientes de incidência, para evitar a sobrecarga e colapso no sistema regional de saúde do Oeste baiano. Destaca-se, a necessidade de uma resposta regional coordenada entre os municípios, a fim de que as decisões e estratégias de enfrentamento da COVID-19 possam ter um alcance mais efetivo. 


\section{Colaboradores}

Os autores contribuíram em todas as etapas do estudo: a) a concepção e o delineamento ou a análise e interpretação dos dados, b) redação do artigo ou a sua revisão crítica, e c) aprovação da versão a ser publicada.

\section{Referências}

1. Huang C, Wang Y, Li X, Ren L, Zhao J, Hu Y, Zhang L, Fan G, Xu J, Gu X, Cheng Z, Yu T, Xia J, Wei Y, Wu W, Xie X, Yin W, Li H, Liu M, Xiao Y, Gao H, Guo L, Xie J, Wang G, Jiang R, Gao Z, Jin Q, Wang J, Cao B. Clinical features of patients infected with 2019 novel coronavirus in Wuhan, China. Lancet 2020; 395(10223):497506.

2. World Health Organization (WHO). Statement on the second meeting of the international health regulations (2005) emergency committee regarding the outbreak of novel coronavirus (2019- $n \mathrm{CoV})$. Genebra: WHO; 2020.

3. Organização Pan-Americana da Saúde (OPAS). Folha informativa - COVID-19 (doença causada pelo novo coronavírus). Brasília: OPAS; 2020.

4. World Health Organization (WHO). WHO announces COVID-19 outbreak a pandemic. Genebra: WHO; 2020.

5. Shi H, Han X, Jiang N, Jiang N, Cao Y, Alwalid O, Gu J, Fan Y, Zheng C. Radiological findings from 81 patients with COVID-19 pneumonia in Wuhan, China: a descriptive study. Lancet Infect Dis 2020; 20(4):425434.

6. Frater JL, Zini G, d'Onofrio G, Rogers HJ. COVID-19 and the clinical hematology laboratory. Int $\mathrm{J} \mathrm{Lab} \mathrm{He}$ matol 2020; 42(Supl. 1):11-18.

7. Hallal PC. Worldwide differences in COVID-19-related mortality. Cien Saude Colet 2020; 25(1):2403-2410.

8. Werneck GL, Carvalho MS. A pandemia de COVID-19 no Brasil: crônica de uma crise sanitária anunciada. Cad Saude Publica 2020; 36(5):e00068820.

9. Cavalcante JR, Abreu AJL. COVID-19 no município do Rio de Janeiro: análise espacial da ocorrência dos primeiros casos e óbitos confirmados. Epidemiol Serv Saude 2020; 29(3):e2020204.

10. Hallal PC, Horta BL, Barros AJD, Dellagostin OA, Hartwig FP, Pellanda LC, Struchiner CJ, Burattini MN, Silveira MF, Menezes AMB, Barros FC, Victora CG. Evolução da prevalência de infecção por COVID-19 no Rio Grande do Sul, Brasil: inquéritos sorológicos seriados. Cien Saude Colet 2020; 25(Supl. 1):2395-2401.

11. Silva JH, Oliveira EC, Hattori TY, Lemos ERS, TerçasTrettel ACP. Descrição de um cluster da COVID-19: o isolamento e a testagem em assintomáticos como estratégias de prevenção da disseminação local em Mato Grosso, 2020. Epidemiol Serv Saude 2020; 29(4):e2020264

12. Freitas ARR, Napimoga M, Donalisio MR. Análise da gravidade da pandemia de Covid-19. Epidemiol Serv Saude 2020; 29(2):e2020119.

13. Floss M, Franco CM, Malvezzi C, Silva KV, Costa BR, Silva VXL, Werreria NS, Duarte DR. A pandemia de COVID-19 em territórios rurais e remotos: perspectiva de médicas e médicos de família e comunidade sobre a atenção primária à saúde. Cad Saude Publica 2020; 36(7):e00108920.

14. Brasil. Ministério da Saúde (MS). Painel Coronavírus - Covid-19 [Internet]. [acessado 2021 maio 4]. Disponível em: https://covid.saude.gov.br/. 
15. Secretaria do Estado da Bahia (SESAB). Boletim Epidemiológico Covid-19 - Bahia, n. 372 [Internet]. 2021 [acessado 2021 maio 4]. Disponível em: http://www. saude.ba.gov.br/wp-content/uploads/2021/03/BOLETIM_ELETRONICO_BAHIAN_372__31032021. pdf.

16. Pedrosa NL, Albuquerque NLS. Análise espacial dos casos de COVID-19 e leitos de terapia intensiva no estado do Ceará, Brasil. Cien Saude Colet 2020; 25(1):2461-2468.

17. Monitora Covid-19. Tendências atuais da pandemia de Covid-19: interiorização e aceleração da transmissão em alguns estados. Nota Técnica [Internet]. 2020 [acessado 2020 ago 25]. Disponível em: https://www.agb. org.br/covid19/wp-content/uploads/2020/06/MonitoraCovid_NotaTecnica_Tend\%C3\%AAncias-atuaisda-pandemia-de-Covid-19-Interiorizacao-e-aceleracao-da-transmissao-em-alguns-estados.pdf.

18. Secretaria de Saúde do Estado da Bahia (SESAB). Regiões de Saúde do estado da Bahia [Internet]. [acessado 2020 ago 20]. Disponível em: http://wwwl.saude. ba.gov.br/mapa_bahia/result_macroch.asp?MA$\mathrm{CRO}=$ OESTE\&Button $122=\mathrm{Ok}$.

19. Instituto Brasileiro de Geografia e Estatística (IBGE). Geociências: Downloads [Internet]. 2010 [acessado 2020 ago 22]. Disponível em: https://www.ibge.gov. br/geociencias/downloads-geociencias.html.

20. Superintendência de Estudos Econômicos e Sociais da Bahia (SEI). Geoinformação: Limites Territoriais [Internet]. 2019 [acessado 2020 ago 22]. Disponível em: https://www.sei.ba.gov.br/index.php?option $=\mathrm{com}_{-}$ content\&view $=$ article\&id=2617\&Itemid=537.

21. Câmera G, Carvalho MS. Análise Espacial de Evento. In: Druck S, Carvalho MS, Câmera G, Monteiro AMV, editores. Análise Espacial de Dados Geográficos. Brasília: Emprapa; 2004. p. 21-52.

22. Kerr L, Kendall C, Silva AAM, Aquino EML, Pescarini JM, Almeida RLF, Ichihara MY, Oliveira JF, Araújo TVB, Santos CT, Jorge DCP, Miranda Filho DB, Santana G, Gabrielli L, Albuquerque MFPM, Almeida-Filho N, Silva NJ, Souza R, Ximenes RAA, Martelli CMT, Brandão Filho SP, Souza WV, Barreto ML. COVID-19 no Nordeste brasileiro: sucessos e limitações nas respostas dos governos dos estados. Cien Saúde Colet 2020; 25(Supl. 2):4099-4120.

23. Brasil. Ministério da Saúde (MS). Boletim epidemiológico especial no 56. Doença pelo Coronavírus COVID-19. Semana epidemiológica 12 (21 a 27/03/2021) [Internet]. [acessado 2021 abr 1]. Disponível em: https://www.gov.br/saude/pt-br/media/pdf/2021/ abril/01/boletim_epidemiologico_covid_56.pdf.

24. Heymann DL, Shindo N. COVID-19: what is next for public health? Lancet 2020; 395(10224):542-545.

25. Croda J, Oliveira WK, Frutuoso RL, Mandetta LH, Baia-da-Silva DC, Brito-Sousa JD, Monteiro WM, Lacerda MVG. COVID-19 in Brazil: advantages of a socialized unified health system and preparation to contain cases. J Brazilian Soc Trop Med 2020; 53:e20200167.
26. Santos MPA, Nery JS, Goes, EF, Silva A, Santos ABS, Batista LE, Araújo EM. População negra e Covid-19: reflexões sobre racismo e saúde. Estud Av 2020; 34(99):225-244.

27. World Health Organization (WHO). Coronavirus disease (Covid-19) advice for the public [Internet]. [acessado 2020 ago 29]. Disponível em: https://www.who. int/emergencies/diseases/novel-coronavirus-2019/ advice-for-public.

28. Goes EF, Ramos DO, Ferreira AJF. Desigualdades raciais em saúde e a pandemia da Covid-19. Trab Educ Saude 2020; 18(3):e00278110.

29. Secretaria do Estado da Bahia (SESAB). Boletim informativo COVID-19: Trabalhadores da saúde [Internet]. [acessado 2020 ago 29]. Disponível em: http://www. saude.ba.gov.br/wp-content/uploads/2020/08/16\%C2\%BA-Boletim-Informativo-COVID-19-TRABALHADORES-DA-SA\%C3\%9ADE.pdf.

30. Instituto Brasileiro de Geografia e Estatística (IBGE). Cidades: Bahia [Internet]. 2017 [acessado 2020 ago 29]. Disponível em: https://cidades.ibge.gov.br/brasil/ $\mathrm{ba} /$ barreiras/pesquisa/38/0.

31. Marinelli NP, Albuquerque LPA, Sousa IDB, Batista FMA, Mascarenhas MDM, Rodrigues MTP. Evolução de indicadores e capacidade de atendimento no início da epidemia de COVID-19 no Nordeste do Brasil, 2020. Epidemiol Serv Saude 2020; 29(3):e2020226.

Artigo apresentado em 20/11/2020

Aprovado em 24/05/2021

Versão final apresentada em 26/05/2021

Editores-chefes: Romeu Gomes, Antônio Augusto Moura da Silva 